\title{
SAPHO SYNDROME: A RARE DISORDER
}

Roberto Claudio de Oliveira Lima Filho' ${ }^{1}$, Oder Banhara Duarte ${ }^{1}$, Rosimeire Mitsuko Tanaka², Flavio Barboza1,*

1.Universidade Federal de Mato Grosso, Sinop (MT); 2.Clínica Byuderme, Sinop (MT), Brazil.

*Corresponding author: flavio.fbarboza@uol.com.br

\section{BACKGROUND}

SAPHO is an acronym for synovitis, acne, pustulosis, hyperostosis and osteitis, regarding manifestations of a rare inflammatory syndrome that affects the skin, joints and bones. The main osteoarticular findings include sternum and unilateral sacroiliac involvement, in addition to peripheral arthritis. The cutaneous manifestations mainly involve palmoplantar pustulosis and severe diffuse acne. Although its pathogenesis is poorly identified, it is estimated that $\mathrm{SAPHO}$ is an autoinflammatory disorder, associated with genetic (13\% of cases are HLA-B27 positive), environmental and immunological factors.

\section{CASE REPORT}

Male, 29 years old, referred with a previous diagnosis of isolated peripheral spondyloarthritis B27 positive, with disabling asymmetric oligoarthritis in the lower limbs for 6 months, using nonsteroidal anti-inflammatory drug (NSAID) and methotrexate. After screening biologic therapy, and one month of isoniazid $300 \mathrm{mg} /$ day for latent tuberculosis (Mantoux $22 \mathrm{~mm}$ ), he starts treatment with anti-lL-17 (this chosen was because the patient works in a COVID-19 area of an intensive care hospital). After first subcutaneous application, he developed severe acneiform rash over the body. Both isoniazid and anti-IL-17 were discontinued, due to the possibility of druginduced hypersensitivity reactions. Unfortunately, the patient began with severe inflammatory low back pain, chest anterior pain and progressive worse in acne (Figure 1). Physical exam revealed positive Patrick's FABER test on the left and arthritis at right wrist and right ankle, proximal left second interphalangeal joint block, severe pain on palpation in costochondral joints and manubrium

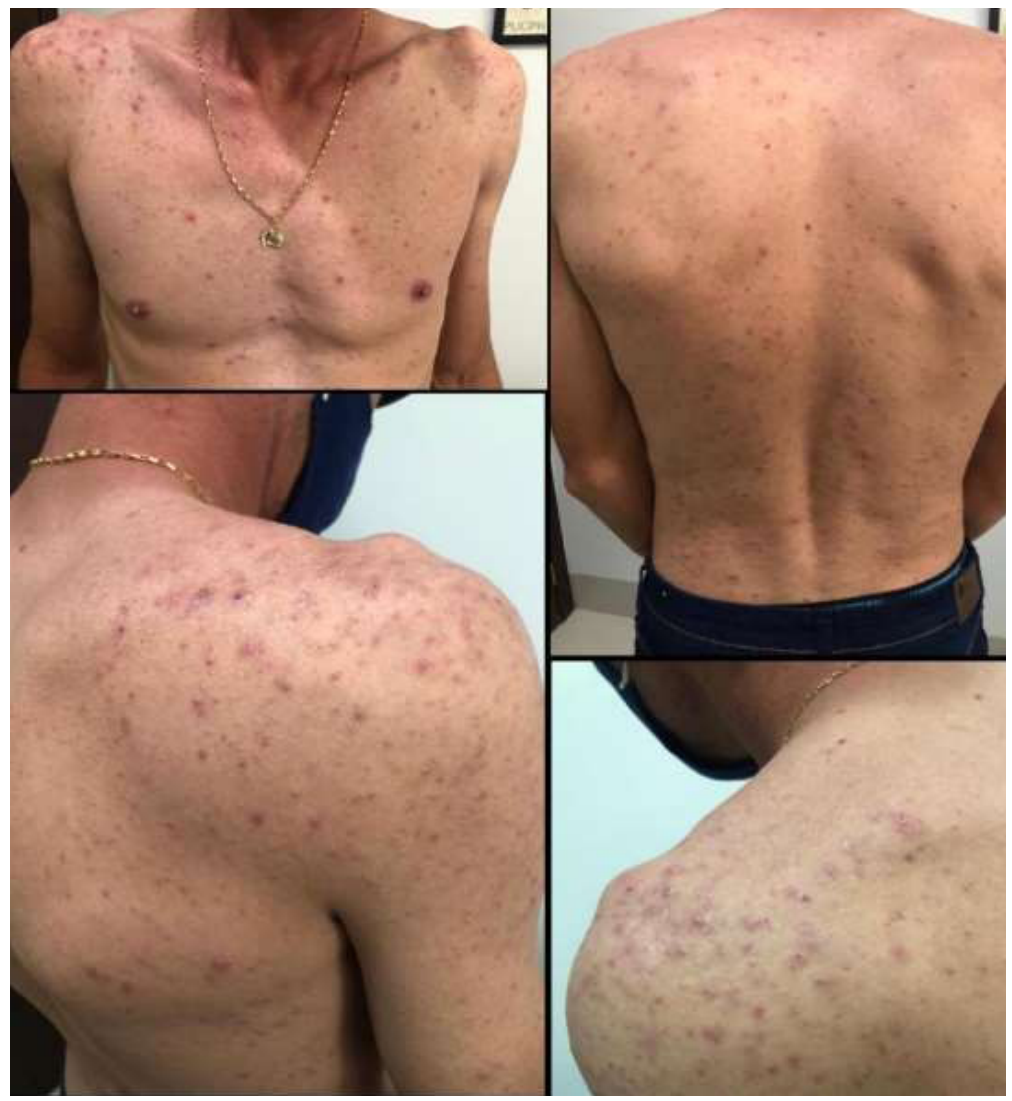

Figure 1. Acneiform eruptions on the body. 
sternal arthritis. The sacroiliac resonance revealed the involvement of unilateral left sacroiliitis. Bone scintigraphy showed osteitis in thoracic region and uptake in the sacroiliac and peripheral joins (Figure 2). Skin biopsy showed suppurative folliculitis compatible with an acneiform eruption, excluding cutaneous manifestations of drug hypersensitivity. After another infectious causes were excluded, the diagnosis of SAPHO syndrome was firmed, and a multidisciplinary treatment with rheumatology and dermatology was started. The therapy with anti-TNF associated with isotretinoin offered a fast and progressive improvement in articular and cutaneous findings after two months of treatment.

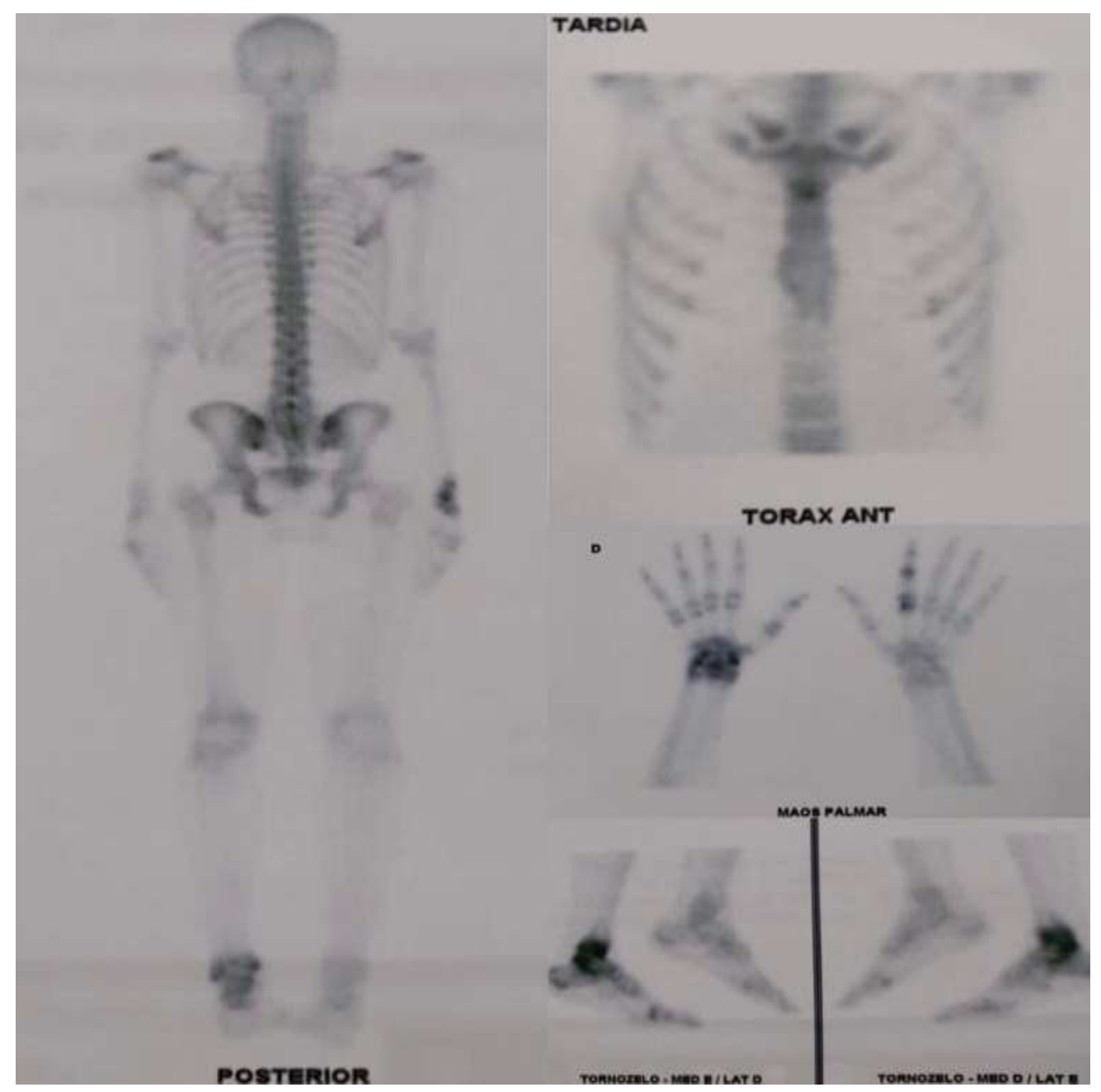

Figure 2. Bone scintigraphy of the patient.

\section{CONCLUSION}

Although it is a condition rarely seen in medical practice, SAPHO syndrome should be part of the differential diagnosis of spondyloarthritis. Furthermore, severe acneiform manifestation should be remembered as a manifestation of the syndrome, and it does not always occur simultaneously with the presentation of the disease, being more common at the first year of the onset of the joint condition. The anterior chest pain with acne should rise suspect of SAPHO syndrome. An association of anti-TNF with isotretinoin has been reported as a good therapeutic option for extensive cutaneous acne and articular symptoms.

\section{KEYWORDS:}

SAPHO syndrome, Spondyloarthritis, Acne, Osteitis. 